\title{
HISTÓRIA ORAL TEMÁTICA NA PESQUISA EM ENFERMAGEM: ESTUDO BIBLIOMÉTRICO*
}

Maria Lúcia Azevedo Ferreira de Macêdo', Maria Cláudia Medeiros Dantas de Rubim Costa1, Simone Pedrosa Lima1, Maria Itayra Padilha², Miriam Süsskind Borenstein²

${ }^{1}$ Enfermeira. Mestre em Enfermagem. Natal-RN-Brasil.

²Enfermeira. Doutora em Enfermagem. Florianópolis-SC-Brasil.

RESUMO: Estudo bibliométrico que objetivou caracterizar a produção científica da enfermagem e a utilização da história oral temática como método ou técnica no contexto brasileiro, de janeiro de 2008 a setembro de 2012, indexada em bases de dados nacionais e internacionais. Utilizou-se para a busca o termo "história oral temática". Selecionaram-se 32 artigos. A maioria dos estudos se concentrou no ano de 2010, nas regiões sudeste e nordeste do País, e as áreas temáticas mais estudadas foram saúde da mulher, educação em enfermagem e saúde mental. Observou-se que os campos de pesquisa da história oral temática são em sua maior parte espaços coletivos, e os sujeitos das pesquisas, muitas vezes, encontram-se vulneráveis em relação a alguma condição instável de saúde e/ou de vida. É um importante caminho científico de dar "vez e voz" às pessoas nos contextos de saúde e na enfermagem.

DESCRITORES: Enfermagem; História; Pesquisa Quantitativa.

\section{THEMATIC ORAL HISTORY IN NURSING RESEARCH: A BIBLIOMETRIC STUDY}

\begin{abstract}
This bibliometric study aimed to characterize the nursing scientific production and the use of thematic oral history as a method or technique in the Brazilian context, between January 2008 and September 2012, indexed in Brazilian and international databases. For the search, the term "thematic oral history" was used. A total of 32 articles was selected. The majority of the studies were concentrated in 2010, in the south-east and north-east regions of Brazil, and the most-studied thematic areas were women's health, education in nursing, and mental health. It was observed that the fields of research in thematic oral history are mainly collective spaces, and the subjects of the studies are often vulnerable in relation to some unstable health and/or life condition. Giving "a time and a voice" to people in the health and nursing contexts is an important scientific path.
\end{abstract}

DESCRIPTORS: Nursing; History; Quantitative Research.

\section{HISTORIAORALTEMÁTICA EN LA INVESTIGACIÓN EN ENFERMERÍA: ESTUDIO BIBLIOMÉTRICO}

RESUMEN: Estudio bibliométrico que tuvo el propósito de caracterizar la producción científica de la enfermería y la utilización de la historia oral temática como método o técnica en el contexto brasileño, de enero de 2008 a septiembre de 2012, indexada en bases de datos nacionales e internacionales. Para la búsqueda, fue utilizado el término "historia oral temática". Treinta y dos artículos fueron seleccionados. La mayoría de los estudios se concentró en el año de 2010, en las regiones sudeste y nordeste del País, y las áreas temáticas más estudiadas fueron salud de la mujer, educación en enfermería y salud mental. Se observó que los campos de investigación de la historia oral temática son, en la mayor parte, espacios colectivos, y los sujetos de las investigaciones, muchas veces, son vulnerables considerando alguna condición instable de salud y/o de vida. Es un importante camino científico para dar "vez y voz" a las personas en los contextos de salud y en la enfermería.

DESCRIPTORES: Enfermería; Historia; Investigación cuantitativa.

*Artigo desenvolvido na disciplina História em Educação, Saúde e Enfermagem do Programa de Pós-Graduação em Enfermagem da Universidade Federal de Santa Catarina.

Recebido: $25 / 04 / 2013$ Finalizado: 19/12/2013

Autor Correspondente:

Maria Lúcia Azevedo Ferreira de Macêdo.

Universidade Federal do Rio Grande do Norte

BR 101, Campus Universitário - 59078-970 - Natal-RN-Brasil

E-mail: mlfmacedo@ig.com.br 


\section{INTRODUÇÃO}

No decorrer da história, as pessoas de um modo geral e principalmente intelectuais, historiadores de ofício, entre outros, buscam compreender as mudanças e as revoluções pelas quais passam em suas próprias vidas. A história oral pode ser um meio pelo qual as pessoas se utilizam para fazer/escrever e contar a história, podendo transformar tanto o conteúdo como a finalidade da história ${ }^{(1)}$.

Em todo mundo, a história oral se destaca por ser um recurso crescente, prático, convincente. Objetiva apreender e registrar experiências e vivências de pessoas e grupos que estão dispostos a testemunhar ou que são convidados para, pela fala, transformar sua experiência em documentos escritos $^{(2)}$. Busca produzir conhecimentos históricos, científicos, e não apenas fazer um relato ordenado da experiência e da vida de outras pessoas ${ }^{(3)}$.

$\mathrm{Na}$ história oral método e técnica têm suas especificidades. Como método privilegia as entrevistas, sendo estas a atenção central dos estudos, focalizando os testemunhos como ponto fundamental das análises, como técnica, supõese que exista uma documentação paralela, e que os depoimentos seriam um recurso adicional. Método é um mecanismo que se configura como um processo sistematizado e rigoroso de pesquisa, e técnica é um conjunto de regras que dirigem os processos de investigações ${ }^{(2)}$.

A história oral temática parte de um assunto específico e a objetividade é direta. As particularidades da história pessoal de quem narra somente interessam na medida em que se identificam aspectos que podem ser úteis à informação temática central ${ }^{(4)}$.

Nesse sentido, pode-se observar que no Brasil, estudos que utilizam o método histórico vêm sendo desenvolvidos por enfermeiros, revelando um pouco do que tem sido a enfermagem brasileira na sua diversidade, seja no que se refere ao início do conhecimento dos próprios enfermeiros sobre sua identidade e importância, bem como do reconhecimento pela sociedade, do profissional enfermeiro, tanto individualmente como no coletivo $^{(5)}$. Percebe-se assim, a importância desse método de pesquisa para compreensão da profissão e sua interface na sociedade.

Este estudo teve por objetivo caracterizar a produção científica da enfermagem na utilização da história oral temática como método ou técnica no contexto brasileiro, no período de janeiro de 2008 a setembro de 2012. A partir dos resultados alcançados, este estudo contribuirá para divulgação da história oral temática como um caminho metodológico de pesquisa na enfermagem.

\section{MÉTODO}

No intuito de alcançar o objetivo desta pesquisa, realizou-se uma bibliometria ${ }^{(6)}$. Inicialmente foi delineado o tema a ser estudado, o objetivo do estudo, o termo a ser utilizado na busca nas bases de dados, e definida a questão de pesquisa: "Como se caracteriza a produção científica da enfermagem que utiliza a história oral temática como método ou técnica no contexto brasileiro no período entre janeiro de 2008 a setembro de 2012?".

A seguir procedeu-se ao levantamento dos artigos disponibilizados em modelo de publicação eletrônica nas bases de dados Latino-Americana e do Caribe de Informação em Ciências da Saúde (LILACS), Scientific Electronic Library Online (SciELO) e Base de dados de enfermagem (BDENF). O acesso às bases de dados deu-se via Biblioteca Virtual em Saúde (BVS), utilizando o termo "história oral temática". Duas revisoras realizaram a busca e a seleção das produções científicas de forma independente buscando garantir a confiabilidade do processo. A coleta de dados ocorreu no mês de outubro de 2012.

Para a seleção dos artigos científicos foi desenvolvida a leitura dos títulos e resumos das publicações que atendiam aos critérios de inclusão estabelecidos: artigos com textos completos disponíveis em suporte eletrônico em português, inglês ou espanhol, bem como estudos, que apresentavam no resumo, o termo "história oral temática", desenvolvidos pela enfermagem, no período compreendido entre janeiro de 2008 e setembro de 2012, realizados no contexto brasileiro. Foram definidos como critérios de exclusão: publicações repetidas nas bases de dados, teses e revisões de literatura.

Levantaram-se 215 resumos, que, após a análise, totalizaram 32 publicações consideradas referentes ao tema em estudo. Na etapa de interpretação, realizou-se uma leitura criteriosa dos artigos, momento em que se fez a discussão dos resultados. 
Por fim, realizou-se a síntese do conhecimento e apresentação da análise dos dados, emergindo o conhecimento atual sobre a utilização da história oral temática como método ou técnica em estudos de enfermagem no contexto brasileiro.

Ressalta-se que princípios éticos permearam o processo de elaboração desse estudo, ao se respeitar a autoria e integridade dos artigos que compuseram essa bibliometria.

\section{RESULTADOS}

Os resultados demonstraram que no ano de 2010 houve um maior número de publicações com
11, seguido dos anos 2009 e 2008 que tiveram o mesmo quantitativo de artigos publicados, sete cada. Quanto às bases de dados, observou-se que a maioria dos estudos está simultaneamente em mais de uma base de pesquisa. Foram encontradas 12 publicações na LILACS/SciELO, nove na LILACS/SCIELO/BDENF, e, seis na LILACS/ BDENF. Quanto ao idioma, 31 estudos foram publicados em português e um em espanhol.

Os Quadros 1, 2 e 3 apresentam as produções científicas da enfermagem brasileira que utilizaram a história oral temática como método ou técnica no período compreendido entre janeiro de 2008 e setembro de 2012.

Quadro1 - Títulos das produções científicas da enfermagem brasileira, região sudeste, que utilizaram a história oral temática como método ou técnica entre os anos 2008 e 2012. Florianópolis, 2013

O cotidiano dos alunos na escola de enfermagem Alfredo Pinto (1949-1956).

Cuidado da enfermidade em casa ou em instituição de saúde: o processo de decisão em uma comunidade de baixa renda.

Significado de ter incontinência urinária e ser incontinente na visão das mulheres.

Vivencias de la paternidad en la adolescencia en una comunidad brasileña de baja renta.

Humanização do cuidado de enfermagem: o que é isso?

Processo de cuidar do idoso em Diálise Peritoneal Ambulatorial Contínua no domicílio.

Atuação do enfermeiro na prevenção e progressão da doença renal crônica.

Percepções e conhecimentos de pacientes com doença renal crônica em tratamento conservador.

A inserção de enfermeiras militares na Força Aérea Brasileira (1981-1982).

Prevenção e progressão da doença renal crônica: atuação do enfermeiro com diabéticos e hipertensos.

Formação e exercício profissional: narrativas de ex-alunas da Escola de Enfermagem.

Atividade de vida diária dos pacientes em tratamento de diálise peritoneal intermitente com cicladora.

Participação das enfermeiras no processo de implantação de práticas obstétricas humanizadas na maternidade Alexander Fleming (1998-2004).

A percepção do enfermeiro, graduado na década de 1990, sobre o seu processo de formação.

Um casal de idosos e sua longa convivência com quatro filhos esquizofrênicos.

Quadro 2 - Títulos das produções científicas da enfermagem brasileira, região sul, que utilizaram a história oral temática como método ou técnica entre os anos 2008 e 2012. Florianópolis, 2013

O portador de transtorno mental e a vida em família.

O itinerário terapêutico: história oral de idosos com câncer.

A autonomia da enfermagem gerontológica no Brasil, segundo as pioneiras (1970-1996).

As estratégias do poder no contexto da maternidade Carmela Dutra: Florianópolis-SC (1956-1986). 
Quadro 3 - Títulos das produções científicas da enfermagem brasileira, região nordeste, que utilizaram a história oral temática como método ou técnica entre os anos 2008 e 2012. Florianópolis, 2013

Práticas terapêuticas na rede informal com ênfase na saúde mental: histórias de cuidadoras.

Infidelidade masculina e violência doméstica: vivência de um grupo de mulheres.

Possibilidades e limites da recuperação do sono de trabalhadores noturnos de enfermagem.

Enfrentamento da AIDS entre mulheres infectadas em Fortaleza - CE.

Escola Maria Antoinette Blanchot e a institucionalização do ensino auxiliar de enfermagem no Piauí.

Centro rural universitário de treinamento e ação comunitária - um resgate histórico 1975-1986.

Abandono do tratamento de tuberculose e relações de vínculo com a equipe de saúde da família.

Saúde mental e enfermagem na estratégia saúde da família: como estão atuando os enfermeiros?

História oral: a experiência das doulas no cuidado à mulher.

Alterações na vida de mulheres com Síndrome de Imunodeficiência Adquirida em face da doença.

Experiências dos enfermeiros com o trabalho de gestão em saúde no estado da Paraíba.

Sentimentos de culpa atribuídos por mulheres com AIDS face a sua doença.

A relação do processo saúde-doença e o trabalho na mineração.

Em relação às revistas científicas observouse que o maior número de publicações ocorreu na revista Texto \& Contexto Enfermagem, Revista do Programa de Pós-Graduação em Enfermagem da Universidade Federal de Santa Catarina, seguida da Revista da Escola de Enfermagem da USP (Universidade de São Paulo), ambas com cinco publicações cada. A Acta Paulista de Enfermagem, órgão oficial de divulgação científica da Escola Paulista de Enfermagem da Universidade Federal de São Paulo, e a Revista Enfermagem UERJ (Universidade Estadual do Rio de Janeiro), publicação da Faculdade de Enfermagem da UERJ, tiveram quatro publicações cada. Quanto às regiões brasileiras onde se efetuaram os estudos, destacam-se a região sudeste com 15 trabalhos e a nordeste com 13 .

A história oral temática foi utilizada nos estudos como método ou como técnica, em que, para coletar os dados os autores utilizaram, na sua grande maioria, a entrevista semi-estruturada. Para a análise dos dados, o método da análise de conteúdo foi o mais empregado. Em seis artigos, além das entrevistas foram analisadas fontes documentais escritas.

A Tabela 1 traz as áreas de estudos da enfermagem que mais utilizaram a história oral temática como método ou técnica. A saúde da mulher se destaca com sete publicações, seguida da educação em enfermagem com cinco, e saúde mental com quatro trabalhos.
Tabela 1 - Áreas temáticas das publicações científicas da enfermagem brasileira que utilizaram a história oral temática como método ou técnica entre os anos 2008 e 2012. Florianópolis, 2013

\begin{tabular}{ll} 
Área temática do estudo & $\mathbf{n}$ \\
\hline Saúde da mulher & 7 \\
\hline Educação em enfermagem & 5 \\
\hline Saúde mental & 4 \\
\hline Identidade profissional & 3 \\
\hline Saúde coletiva & 3 \\
\hline Saúde do trabalhador & 2 \\
\hline Saúde do adulto & 2 \\
\hline Processos de enfermagem & 2 \\
\hline Saúde do idoso & 2 \\
\hline Gestão em saúde & 1 \\
\hline Saúde do adolescente & 1 \\
\hline Total & 32 \\
\hline
\end{tabular}

\section{DISCUSSÃO}

O fato dos estudos ocorrerem em maior número na região sudeste pode estar relacionado ao surgimento e fortalecimento da história oral nessa região do País. Em relação ao surgimento, na década de 1970, a Fundação Ford juntamente com a Fundação Getúlio Vargas, Rio de Janeiro, tentou, sem alcançar êxito, devido à ausência de um contexto democrático, estruturar uma organização de alcance nacional. Desse esforço resultou o programa CPDOC/FGV/RJ, pioneiro 
da história oral brasileira. No que se refere ao fortalecimento, em 1992, durante o Congresso Internacional América 92: Raízes e Trajetórias, em São Paulo, no Departamento de História da USP, foi proposta a criação da Associação Brasileira de História Oral $(\mathrm{ABHO})$, que foi efetivada em $1994^{(4)}$.

Os dados dessa pesquisa evidenciam que há um alargamento da utilização da história oral temática como método ou técnica na enfermagem em outras regiões do País, nesse estudo, na região nordeste.

O campo de ação da história oral se amplia não se limitando aos domínios dos historiadores e demais cientistas sociais, visto que ela é também empregada por alguns grupos sociais que tem interesse em construir suas próprias versões de seu acontecer histórico ${ }^{(3)}$.

No que diz respeito às áreas encontradas nos estudos, a área da saúde da mulher demonstrou uma concentração em relação à infecção causada pelo HIV/AIDS em mulheres e as repercussões nas suas vidas ${ }^{(7-9)}$, seguido de estudos que abordaram a importância da doula no acompanhamento ao parto ${ }^{(10)}$, e mulheres vítimas de violência ${ }^{(11)}$, histórico de implantação de práticas obstétricas no contexto de uma maternidade ${ }^{(12)}$ e as consequências da incontinência urinária na vida de mulheres ${ }^{(13)}$.

Os temas apresentados reforçam e estão em consonância com as políticas públicas brasileiras. Os princípios da Política Nacional de Atenção Integral à Saúde da Mulher enfatizam que as mulheres devem ser atendidas em todas as suas especificidades e ciclos de vida, preocupandose com as diferentes faixas etárias e distintos grupos sociais. Assim, os documentos editados pela Política descrevem os componentes dentre os quais se encontra a garantia da assistência ao parto, enfrentamento da violência contra as mulheres e $\operatorname{AIDS}^{(14)}$.

Quanto à AIDS em mulheres, o Ministério da Saúde tem demonstrado preocupação com a transmissão vertical do HIV/AIDS, devido a um aumento proporcional de casos da doença em relações heterossexuais, principalmente entre as mulheres. Esta realidade traz a possibilidade de aumentar o número de crianças contaminadas, se não houver um cuidado específico durante o pré-natal, parto e puerpério(15). A vulnerabilidade à adesão quanto à profilaxia na transmissão vertical tem causado preocupação. Nesse sentido, o enfermeiro deverá estimular espaços dialógicos a fim de esclarecer a população acerca do $\operatorname{HIV}_{\text {AIDS }}{ }^{(16)}$.

A progressiva aceitação da história oral pela história universitária nos últimos anos está relacionada ao aumento das curiosidades do historiador. Nesse contexto, o crescente interesse pela história das mulheres desempenhou importante papel, quer se trate do trabalho, da militância, da vida cotidiana, ou, de sua identidade ou de sua vida afetiva(17).

No que se refere à área da educação em enfermagem destacam-se estudos referentes ao histórico de escolas de enfermagem ${ }^{(18-19)}$, a formação do enfermeiro ${ }^{(20-21)}$, e ao resgate histórico de um centro rural universitário ${ }^{(22)}$.

A história se refaz a partir de novas perguntas elaboradas pelo historiador ou pela descoberta de outras fontes ou documentos. A elaboração da história está voltada para o que se expressou. Essa manifestação ao ser tratada historicamente alcança uma dimensão cronológica ou mesmo temática. A história opera sempre com o que está dito em algum momento, em algum lugar. Desses elementos, o historiador constrói sua narrativa ${ }^{(23)}$.

Quando se busca conhecer a história da enfermagem é que se percebe quanto esta não se separa de outras atividades da vida e do mundo da saúde, e que, realizar a pesquisa histórica da enfermagem é um desafio crescente a ser enfrentado ${ }^{(5)}$.

O uso da história oral é perfeitamente aplicável quando o objeto de estudo são as lacunas que o tempo deixa acerca das experiências vivenciadas por um grupo específico como é o caso dos trabalhos que abordaram os levantamentos da história da enfermagem. Estes visam estabelecer um elo entre passado e presente almejando a construção do futuro ${ }^{(24)}$.

No tocante à área de saúde mental os temas abordados foram: saúde mental e família ${ }^{(25-26)}$, atenção à saúde mental na Estratégia da Saúde da Família ${ }^{(27)}$, e, práticas terapêuticas na rede informal com ênfase na saúde mental ${ }^{(28)}$. As publicações analisadas parecem estar indo ao encontro às diretrizes da reforma psiquiátrica que propõe a redução de leitos psiquiátricos no País e inclusão de ações na rede básica de saúde ${ }^{(29)}$. 
Por sua proximidade com famílias e comunidades, as equipes da Atenção Básica se apresentam como um recurso estratégico para o enfrentamento de importantes problemas de saúde pública, como os agravos vinculados ao uso abusivo de álcool, drogas e diversas outras formas de sofrimento psíquico ${ }^{(29)}$.

Dessa forma, o Ministério da Saúde vem estimulando ativamente, nas políticas de expansão, formulação e avaliação da Atenção Básica, diretrizes que incluam a dimensão subjetiva dos usuários e os problemas mais frequentes de saúde mental ${ }^{(29)}$.

A história oral pode ser empregada para modificar o enfoque da própria história, desvendar novas áreas de investigação; permite devolver às pessoas que vivenciaram a história um lugar fundamental, a partir de suas próprias palavras; e trazer à tona as experiências daquelas pessoas, cuja existência é habitualmente esquecida ${ }^{(1)}$.

Assim, acredita-se que a relevância dos estudos nessas áreas retrata o estímulo dado ao tema e a necessidade de entender através da história do indivíduo e da coletividade a implantação, desenvolvimento e avaliação das políticas públicas.

\section{CONSIDERAÇÕES FINAIS}

Os estudos publicados nos últimos anos retratam a importância da história oral no esclarecimento de um fenômeno. Assim, esta pesquisa traz uma reflexão sobre a importância dessas publicações para a construção do conhecimento, utilizando-se para tanto a história oral temática como método ou técnica de pesquisa capaz de compreender a construção de processos de cuidado, de formação, de vida e comunidade assistida pela enfermagem.

Nos artigos analisados houve uma predominância por temas referentes à saúde da mulher (garantia de assistência ao parto, AIDS e violência), à educação em enfermagem (formação da enfermagem e históricos de escolas de enfermagem) e à saúde mental (vivência de famílias com a doença mental e práticas terapêuticas), demonstrando que estas áreas retratam uma afinidade com o método ou a técnica da história oral temática, que é um meio de busca de esclarecimentos de experiências vividas sob diferentes situações, portanto pertinentes às referidas áreas.

Observou-se neste estudo, que os campos de pesquisa da história oral temática na enfermagem, seja ela utilizada como método ou como técnica, são, em sua maior parte, espaços coletivos, e os sujeitos das pesquisas, muitas vezes, encontramse vulneráveis em relação a alguma condição difícil de saúde e/ou de vida. Assim, esses trabalhos tornam-se relevantes como marco para o esclarecimento de fenômenos em prol da melhoria do cuidado da enfermagem, bem como propicia que os sujeitos sintam-se inseridos no processo histórico, capazes de refletir e transformar a realidade, olhando para si mesmos e consequentemente tornando-se sujeitos ativos na elucidação desses fenômenos. É um importante caminho científico de dar "vez e voz" às pessoas que continuamente constroem o saber e o fazer nos contextos de saúde na área da enfermagem.

\section{REFERÊNCIAS}

1. Thompson P. A voz do passado: história oral. $2^{\mathrm{a}}$ ed. Rio de Janeiro: Paz e Terra; 1992.

2. Meihy JCSB, Holanda F. História oral: como fazer, como pensar. São Paulo: Contexto; 2011.

3. Lozano JEA. Prática e estilos de pesquisa na história oral contemporânea. In: Amado J, Ferreira MM, organizadores. Uso \& abusos da história Oral. $8^{\mathrm{a}}$ ed. Rio de Janeiro: FGV; 2006.

4. Meihy JCSB. Manual de história oral. $4^{\mathrm{a}}$ ed. São Paulo: Loyola; 2002.

5. Padilha MICS, Borenstein MS. O método de pesquisa histórica na enfermagem. Texto Contexto Enferm. [Internet] 2005;14(4) [acesso em 02 out 2012]. Disponível: http://dx.doi.org/10.1590/S010407072005000400015

6. Araújo CAA. Bibliometria: evolução histórica e questões atuais. Em Questão. [Internet] 2006;12(1) [acesso em 01 out 2012]. Disponível: http://revistas.univerciencia.org/ index.php/revistaemquestao/article/viewFile/3707/3495.

7. Carvalho CML, Galvão MTG. Enfrentamento da AIDS entre mulheres infectadas em Fortaleza - CE. Rev Esc Enferm USP. [Internet] 2008;42(1) [acesso em 03 out 2012]. Disponível: http://dx.doi.org/10.1590/S008062342008000100012

8. Carvalho $\mathrm{CML}$, Galvão MTG. Sentimentos de culpa atribuídos por mulheres com aids face a sua doença. Rev Rene. [Internet] 2010;11(2) [acesso em 02 out 
2012]. Disponível: http://www.revistarene.ufc.br/ revista/index.php/revista/article/view/379/pdf.

9. Carvalho CML, Galvão MTG, Silva RM. Alterações na vida de mulheres com Síndrome de Imunodeficiência Adquirida em face da doença. Acta Paul. Enferm. [Internet] 2010;23(1) [acesso em 30 out 2012]. Disponível: http://dx.doi.org/10.1590/S010321002010000100015 10. Souza KRF, Dias MD. História oral: a experiência das doulas no cuidado à mulher. Acta Paul. Enferm. [Internet] 2010;23(4) [acesso em 30 out 2012]. Disponível: http://dx.doi. org/10.1590/S0103-21002010000400008

11. Trindade CRF, Almeida AM, Alves Rozendo C. Infidelidade masculina e violência doméstica: vivência de um grupo de mulheres. Cienc. enferm. [Internet] 2008;14(2) [acesso em 30 out 2012]. Disponível: http:// dx.doi.org/10.4067/S0717-95532008000200006

12. Progianti JM, Porfirio AB. Participação das enfermeiras no processo de implantação de práticas obstétricas humanizadas na maternidade Alexander Fleming (1998-2004). Esc. Anna Nery. [Internet] 2012;16(3) [acesso em 30 out 2012]. Disponível: http://dx.doi. org/10.1590/S1414-81452012000300003

13. Borba AMC, Lelis MAS, Bretas ACP. Significado de ter incontinência urinária e ser incontinente na visão das mulheres. Texto Contexto Enferm. [Internet] 2008;17(3) [acesso em 28 out 2012]. Disponível: http://dx.doi. org/10.1590/S0104-07072008000300014

14. Ministério da Saúde (BR). Secretaria de Gestão Estratégica e Participativa. Saúde da mulher: um diálogo aberto e participativo. Brasília: Ministério da Saúde; 2010.

15. Ministério da Saúde (BR). Secretaria de Vigilância em Saúde. Programa Nacional de DST e AIDS. Protocolo para prevenção de transmissão vertical de HIV e sífilis. Brasília: Ministério da Saúde; 2007.

16. Langendorf TF, Padoin SMM, Paula CC, Silva CB. Vulnerabilidade na adesão à profilaxia da transmissão vertical do HIV. Cogitare enferm. [Internet] 2012;17(4) [acesso em 12 out 2012]. Disponível: http://ojs. c3sl.ufpr.br/ojs2/index.php/cogitare/article/view File/30388/19663.

17. Joutard P. História oral: balanço da metodologia e da produção nos últimos 25 anos. In: Amado J, Ferreira, MM, organizadores. Usos \& abusos da história oral. $8^{\mathrm{a}}$ ed. Rio de Janeiro: FGV; 2006. p. 42-63.

18. Meirelles MR, Amorim WM. O cotidiano dos alunos na escola de enfermagem Alfredo Pinto (1949-1956). Rev. Latino-Am. Enfermagem. [Internet] 2008;16(6) [acesso em 12 out 2012]. Disponível: http://dx.doi. org/10.1590/S0104-11692008000600011

19. Vilar BM, Borges LDVNM, Santos AMR. Escola
Maria Antoinette Blanchot e a institucionalização do ensino auxiliar de enfermagem no Piauí. Rev. bras enferm. [Internet] 2008;61(5) [acesso em 10 out 2012]. Disponível: http://dx.doi.org/10.1590/S003471672008000500019

20. Carrijo AR, Oguisso T, Campos PFS. Formação e exercício profissional: narrativas de ex-alunas da Escola de Enfermagem Lauriston Job Lane. R. Pesq.: cuid. fundamental. [Internet] 2010;2(2) [acesso em 10 out 2012]. Disponível: http://www.seer.unirio.br/index. php/cuidadofundamental/article/view/587/pdf_21.

21. Santos $S M R$. A percepção do enfermeiro, graduado na década de 1990, sobre o seu processo de formação. Rev. enferm. UERJ. [Internet] 2011;19(4) [acesso em 15 out 2012]. Disponível: http://www.facenf.uerj.br/ v19n4/v19n4a07.pdf.

22. Leite MTF, Nunes BMVT. Centro rural universitário e ação comunitária: um resgate histórico 1975-1986. Texto Contexto Enferm. [Internet] 2009;18(3) [acesso em 06 out 2012]. Disponível: http://dx.doi.org/10.1590/ S0104-07072009000300004

23. Montenegro AT. História oral e memória: a cultura popular revisitada. 5a ed. São Paulo: Contexto; 2003.

24. Padilha MICS, Borenstein MS. História da enfermagem: ensino, pesquisa e interdisciplinaridade. Esc. Anna Nery. [Internet] 2006;10(3) [acesso em 06 out 2012]. Disponível: http://dx.doi.org/10.1590/S141481452006000300024

25. Brischiliari A, Waidman MAP. O portador de transtorno mental e a vida em família. Esc. Anna Nery. [Internet] 2012;16(1) [acesso em 04 out 2012]. Disponível: http:// dx.doi.org/10.1590/S1414-81452012000100020

26. Oliveira RM, Furegato ARF. Um casal de idosos e sua longa convivência com quatro filhos esquizofrênicos. Rev Esc Enferm USP. [Internet] 2012;46(3) [acesso em 18 out 2012]. Disponível: http://dx.doi.org/10.1590/ S0080-62342012000300013

27. Ribeiro LM, Medeiros SM, Albuquerque JS, Fernandes SMBA. Saúde mental e enfermagem na estratégia saúde da família: como estão atuando os enfermeiros? Rev Esc Enferm USP. [Internet] 2010;44(2) [acesso em 9 out 2012]. Disponível: http://dx.doi.org/10.1590/ S0080-62342010000200019

28. Saraiva AM, Ferreira Filha MO, Dias MD. Práticas terapêuticas na rede informal com ênfase na saúde mental: histórias de cuidadoras. Rev. Eletr. Enf. [Internet] 2008;10(4) [acesso em 3 out 2012]. Disponível: http://www.fen.ufg.br/fen_revista/v10/n4/ pdf/v10n4a12.pdf.

29. Ministério da Saúde (BR). Secretaria de Atenção à Saúde. Coordenação Geral de Saúde Mental. Reforma psiquiátrica e política de saúde mental no Brasil. 
Documento apresentado à Conferência Regional de Reforma dos Serviços de Saúde Mental: 15 anos depois de Caracas. Brasília: Ministério da Saúde; 2005. 